\title{
COMPUTATIONAL APPROACHES FOR THE PREDICTION OF ANTIMICROBIAL POTENTIAL PEPTIDES FROM OCIMUM TENUIFLORUM
}

\author{
SUNIL KUMAR SURYAWANSHI*, USHA CHOUHAN
}

Department of Bioinformatics, Maulana Azad National Institute of Technology, Bhopal, Madhya Pradesh, India. Email: Bioinfosunil@gmail.com

Received: 10 October 2017, Revised and Accepted: 25 November 2017

\section{ABSTRACT}

Objective: In this study, antimicrobial activity was predicted against novel antimicrobial target 1SDE receptor to understand the structural feature of predicted peptides using machine learning approach from Ocimum tenuiflorum.

Methods: Protein sequences from 0 . tenuiflorum were digested using peptide cutter and further processed for the prediction of antimicrobial peptide (AMP) through AMP predictor tool of CAMP which have multidimensional algorithms such as support vector machine, artificial neural network, random forest, and discriminant analysis. After analyzing various peptides, only four peptides were predicted as antimicrobial in nature. Furthermore, the three-dimensional structure of different potential peptides was generated with the help of Pepfold-3.0 server followed by protein-peptide docking studies with novel target receptor with the help of PatchDock, FireDock webserver, and Hex 8.0 software. Interactions were further visualized using Discovery Studio Client 2.5 software tool.

Results: This study revealed that peptide 2 resulted higher score in PatchDock and FireDock and also Hex 8.0 provides E total value of -430.18 which is higher than that of peptide 1 with -381.07 , peptide 3 with -416.86 , and peptide 4 with -407.94 .

Conclusion: The peptide predicted in this study has potential to act as effective AMP against target receptor and also utilize other antimicrobial target.

Keywords: Antimicrobial peptides, artificial neural network, discriminant analysis, non-antimicrobial peptide, random forest, support vector machine.

(C) 2018 The Authors. Published by Innovare Academic Sciences Pvt Ltd. This is an open access article under the CC BY license (http://creativecommons. org/licenses/by/4. 0/) DOI: http://dx.doi.org/10.22159/ajpcr.2018.v11i1.23008

\section{INTRODUCTION}

Excessive use of antibiotics against pathogenic microorganism has lot of side effects as well as drawback. Due to this, potential research in search of alternative options is required to combat with the resistance of bacteria [1,2]. Peptides research is one of the promising areas in the field of medicinal chemistry due to its diverse nature and activity against resistant microorganism, this field of research is highlighted in the current scenario. Peptides are considered as highly selective, efficacious, safe, and well tolerable. Peptidomimetics dataset provides challenges to the scientific community to extract significant information from these generated short peptides. Therefore, nowadays in the pharmaceutical research and development of numerous peptide therapeutics are being evaluated under clinical trials $[3,4]$.

In modern era, medicinal plant is considered as primary source to find potential therapeutics values from its various parts including the seeds, root, stem, and leaves. In this study, we are selected the plant species for our research work is Ocimum tenuiflorum, commonly known as Tulsi, due to its significant medicinal properties including analgesics, antiemetics, and immunomodulatory agents. Previous research concluded that tulsi has strong antibacterial property against various pathogenic Gram-negative bacteria [5,6]. Nanotechnology also utilizes the medicinal properties of this plant and synthesis of nanoparticles from leaves aqueous extract were performed strong antibacterial activity against Gram-positive, Gram-negative, and spore-forming bacteria [7]. To achieve our goal, we utilize protein data from the National Center for Biotechnology Information (NCBI) which is a series of databases relevant to biotechnology and biomedicine.

Integrity of bacteria is well maintained by the bacterial cell wall and $\beta$-lactam-based antibiotics interfere in the biosynthetic pathway of cell wall formation. D-alanyl-D-alanine carboxypeptidase/transpeptidases
(DD-peptidases) are enzymes classified under penicillin-binding protein which catalyze the final transpeptidation reaction of bacterial cell wall [8]. However, we consider DD peptidase as potential therapeutic target site to interact with novel peptide which inhibits the formation of bacterial cell wall.

In the past decades, research work has been done on the inhibition of DD peptidase with lactam antibiotics such as penicillins and cephalosporins [9]. In light of evolution, bacterial resistance has been enhanced due to this antibiotics are no more effective, so here, we approach modeling of peptide therapeutics to inhibit therapeutic target, i.e., DD peptidase (1SDE). In this paper, we presented machine learning based peptide design from 0 . tenuiflorum, which will further modeled to act as ligand molecules. Interaction of predicted peptides 1-4 with target receptor was performed using bioinformatics tools to find out potential and effective novel therapeutics peptide.

\section{METHODS}

Data set

To achieve our goal and develop our methodology, we obtained the dataset of protein sequence, i.e., 0 . tenuiflorum from NCBI search engine with the keyword "tulsi." In number, 30 protein sequences retrieved from NCBI.

\section{In silico proteolysis of $O$. tenuiflorum proteins}

In silico proteolysis of tulsi proteins was subjected to digestion using Expasy-PeptideCutter tool [10]. In this program, chemical and enzymatic option available to digest these proteins, here, we select only enzymatic option due to its specificity. Before processing of site-specific cleavage of protein to peptides, we randomly selected three enzymes, i.e., trypsin, protein kinase, and pepsin. 
Prediction of antimicrobial peptides (AMP) from peptides

Fragmented peptides were subjected to predict antimicrobial nature of peptide sequences with the help of AMP predictor tools available at CAMP database. AMP predictor tool implemented with the four different multivariate statistical methods support vector machines, random forest, artificial neural network (ANN), and discriminant analysis to classify these peptides into two categories, i.e., AMP and non-AMP (NAMP). Development and evaluation of model has been described in detail by Waghu et al. Except ANN, prediction score is in numeric form as a resultant and peptides are classified as AMPs or NAMPs [11]. When each statistical method found peptides as AMP, only after this we scrutinized it as AMP for further experimental process. Modeling three-dimensional (3D) structure of peptide fragments from O. tenuiflorum was predicted by Pepfold-3 webserver [12]. It is based on homology modeling method. This predicted 3D peptide structure act as potential ligand molecules for molecular docking process.

Protein-peptide docking using PatchDock and FireDock webserver We apply PatchDock [13] and FireDock [14] webserver for the molecular docking of peptides 1-4. First, PatchDock is an algorithm, which is categorized into three major steps as (1) molecular shape representation, (2) surface patch matching, and (3) filtering and scoring. Computational simulation of protein-peptides was performed efficient unbound docking of rigid molecules [15]. Second, FireDock webserver plays a key role in the refinement of protein-peptides docking. This method applies on the solution obtained from the fast rigid-body docking [16].

\section{Protein-peptide docking and visualization}

In addition to PatchDock and FireDock, Hex program was also used herein molecular docking which formed intermolecular interactions between receptor and peptide act as ligand. Spherical polar Fourier applied in this program to correlates and accelerate the calculations. We used Hex 8.0 software for rigid docking to compute possible interaction of different peptides with 1sde receptor on the interaction site [17]. Output of the docking was refined using Discovery Studio Client 2.5 software [18]. Further, analysis of docked complex has been performed with the help of Discovery Studio Client 2.5 to perform interaction; peptide 1 and 2 binds to 1 SDE protein receptor.

\section{RESULTS AND DISCUSSION}

\section{In silico proteolysis of 0 . tenuiflorum proteins}

In the initial stage, preprocessing of protein sequences from o. tenuiflorum was performed to predict potential AMP using AMP prediction tool available at CAMP server. Using the knowledge of sitespecific enzymatic cleavage of protein, we implemented three different proteolytic enzymes which provide 420 fragments as resultant. After removing the redundancy among sequences, only 225 non-redundant peptide sequences are screen out. Applying machine learning algorithms of CAMP server on 225 peptides as a resultant only four sequences provide potential antimicrobial properties out of the bunch. Previously, same kind of work has been carried out to prove the efficacy of peptides to being as antimicrobial from different sources [19].

\section{Modeling of peptide using Pepfold 3.0 server}

Pepfold 3.0 server is very flexible software package which utilized by various researchers for protein-peptide docking purpose. In this work for the ligand preparation, Pepfold 3.0 server was used to generate 3D structure from manually curated linear AMPs. This server generates 3D structure from linear sequences of peptides. These 3D structures of peptide act as ligand molecules to be dock with protein receptor. Protein-peptide docking provides new horizon in which peptide drugs were replaced by traditional chemical drugs. As per the significance of antibiotics, there is also drawback with various antibiotics to show deleterious side effects simultaneously, in this paper, we are working on predicted peptides which are originated from natural proteins of o. tenuiflorum.j
Implementation of various molecular docking simulation

Docking is a prediction method where peptide residue binds with the target receptor. To implement this method, many protein-peptide docking tools, i.e., PatchDock, FireDock, and Hex 8.0 are often employed. Four different peptide models (1-4) docked here, and next, to identify possible binding sites during blind docking simulation with the help of visualization tool. The docking complex is shown in Fig. 1a-d.

\section{Analysis of docked complex}

The peptide-receptor complex further analyzed by Discovery Studio 2.5 to determine the binding sites residues. The residues play major role in efficient binding manner is shown in Table 1 .

Molecular docking score of all the peptides with receptor 1SDE using different techniques is shown in Table 2. Using PatchDock server, we found that peptide 2 has highest score, area, and angiotensin-converting enzyme (ACE) values of 10,048, 1547.60, and -108.89 , respectively. On the basis of shape complementarity of soft molecular surfaces, best starting candidate solutions were generated by PatchDock algorithms. After that, optimization, refinement of top 10 candidate solutions was performed by FireDock, wherein the restriction of flexibility to the side chains of interacting surface and small rigid body movements are allowed to score value of global energy, attractive VdW, repulsive VdW, ACE, and HB, i.e., $-36.41,-18.41$, and 5.05, respectively, for peptide 2 was found best among rest of other peptides. Finally, protein-peptide docking software Hex 8.0 provides docking result in terms of global energy of peptide 2 is -430.18 which highest than $-381.07,-416.86$, and -407.94 of peptide 1 , peptide 3 , and peptide 4 , respectively. Hex 8.0 software is the most implemented tool to dock proteins with peptide, so we are focusing on the best outcomes of our result of peptide 2 with receptor.

The $\mathrm{H}$ atom of Tyr 137 residue of peptide 1 is found to be interacting with the oxygen atom

Valine 5 residue of the receptor-binding pocket. Furthermore, the 0 atom of this 200 residue is found interacting with Ala 12 residue by means of hydrogen-bonding interactions as shown in Fig. 1a. The tyrosine 296 residue of peptide 2 is showing extensive $\mathrm{H}$-bond interaction with the oxygen atom of Lys 30 residue. Furthermore, the terminal $\mathrm{H}$ atom of the Lue3, Trp271, Lys313, Asp314, and Lys316 is found interacting with the oxygen atom of Ile21, Ile10, Ile11, Asn29T, and Asn21, respectively, by means of H-bonding as shown in Fig. $1 \mathrm{~b}$. The molecular interaction of peptide 3 is shown in Fig. 1c that hydrogen of Ala253 interacts with oxygen of Lys16 and oxygen of Gln245 from peptide 4 interact with hydrogen of Val1 of receptor. Hydrogen bonding and other electrostatic interactions between peptide 2 and receptor are found highest as compared to other peptide 1, 3, and 4. Docking prediction dictates here as peptide 2 is strongly interact with target receptor in relative orientation and inhibiting the formation of bacterial cell wall. Peptide 2 interact with receptor, finally, overall eight amino acid of protein interact with eight amino acid residue of peptide. In light of interaction binding of Tyr 296 hydrogen bonded with Lys 30 of peptide at four different sites, i.e., $\mathrm{HH}-01, \mathrm{HH}-\mathrm{O} 2, \mathrm{OH}-01$, and $\mathrm{OH}-02$. In the same way, Asp 314 of protein hydrogen bonded with Asn 29, i.e., H - OD1 and OD1 - HD22. Leu 3 with Ile 24, i.e., H1 - O. Trp 271 with Ile HE1 - 0. Lys 313 with Ile 11, i.e., HZ1 - 0. Lys 316 with Asn 21, i.e., HZ3 - O. Cys 291 with Tyr 27, i.e., O - HH. Ser 312 with Asn 29, i.e., $\mathrm{OG}-\mathrm{HD} 22$.

\section{CONCLUSION}

In the present study, we had concluded that peptides 2 predicted from o. tenuiflorum have properties of being antimicrobial in nature. Our studies concluded that peptide 2 has potential probability to being active AMP due to its highest score by PatchDock, FireDock, and Hex 8.0 tools. As compared to other peptides better results provided by peptide 2 , due to this reason, we strongly recommend this peptide sequence for wet lab validation and its further future aspects. 
Table 1: Molecular interaction between predicted peptide and target receptor

\begin{tabular}{|c|c|c|c|}
\hline Receptor & Predicted peptides & Receptor residue (amino acid) & Ligand residue (amino acid) \\
\hline \multirow[t]{22}{*}{ DD-Peptidase (1SDE) } & Peptide 1 & TYR 137: H & VAL 5: 0 \\
\hline & LDTLVPIIPLIAK & HIS 200: 0 & ALA 12: 01 \\
\hline & & HIS 200: 0 & ALA 12: 02 \\
\hline & Peptide 2 & LEU 3:H1 & ILE 24: 0 \\
\hline & AGDVVGVGCIIGSCGNCRPCNSDIEQYCNK & TRP 271: HE1 & ILE 10: 0 \\
\hline & & TYR $296: \mathrm{HH}$ & LYS 30: 01 \\
\hline & & TYR 296: HH & LYS 30: 02 \\
\hline & & LYS 313: HZ1 & ILE 11: 0 \\
\hline & & ASP 314: H & ASN 29: OD1 \\
\hline & & LYS 316: HZ3 & ASN 21: 0 \\
\hline & & CYS 291: 0 & TYR 27: HH \\
\hline & & SER 312: OG & ASN 29: HD22 \\
\hline & & TYR 296: OH & LYS 30: 01 \\
\hline & & TYR 296: OH & LYS 30: 02 \\
\hline & & ASP 314: OD1 & ASN 29: HD22 \\
\hline & ILVNAWWLANNPAQWK & GLN 258: HE22 & LYS 16: 01 \\
\hline & & GLY 34: 0 & TRP7: HE1 \\
\hline & & SER 252: 0 & LYS 16: 01 \\
\hline & & SER 256: 0 & LYS 16: 01 \\
\hline & & THR 249: 0 & LYS 16: 02 \\
\hline & Peptide 4 & GLN 245: HE22 & VAL1: 0 \\
\hline & VSVAAVVPPLVLALAK & GLN 245: OE1 & VAL1: H2 \\
\hline
\end{tabular}

Table 2: Molecular Docking score of peptide 1-4 with 1SDE receptor by means of different open software

\begin{tabular}{|c|c|c|c|c|c|c|c|c|c|}
\hline \multirow[t]{2}{*}{ Predicted Peptides } & \multicolumn{4}{|c|}{ PatchDock } & \multicolumn{3}{|c|}{ FireDock } & & \multirow{2}{*}{$\begin{array}{l}\text { Hex } 8.0 \\
\text { Global energy }\end{array}$} \\
\hline & Score & Area & ACE & Global energy & Attractive VdW & Repulsive VdW & ACE & HB & \\
\hline Peptide 1 & 8582 & 1001.70 & -127.0 & -33.60 & -18.21 & 3.59 & -7.19 & -0.90 & -381.07 \\
\hline Peptide 2 & 10048 & 1547.60 & -108.89 & -36.41 & -18.41 & 5.05 & -4.59 & -5.61 & -430.18 \\
\hline Peptide 3 & 8280 & 1092.20 & -26.71 & -28.63 & -16.20 & 9.11 & -6.43 & -1.31 & -416.86 \\
\hline Peptide 4 & 7864 & 936.30 & -105.28 & -34.86 & -14.02 & 6.49 & -10.99 & -1.56 & -407.94 \\
\hline
\end{tabular}

ACE: Atomic Contact Energy
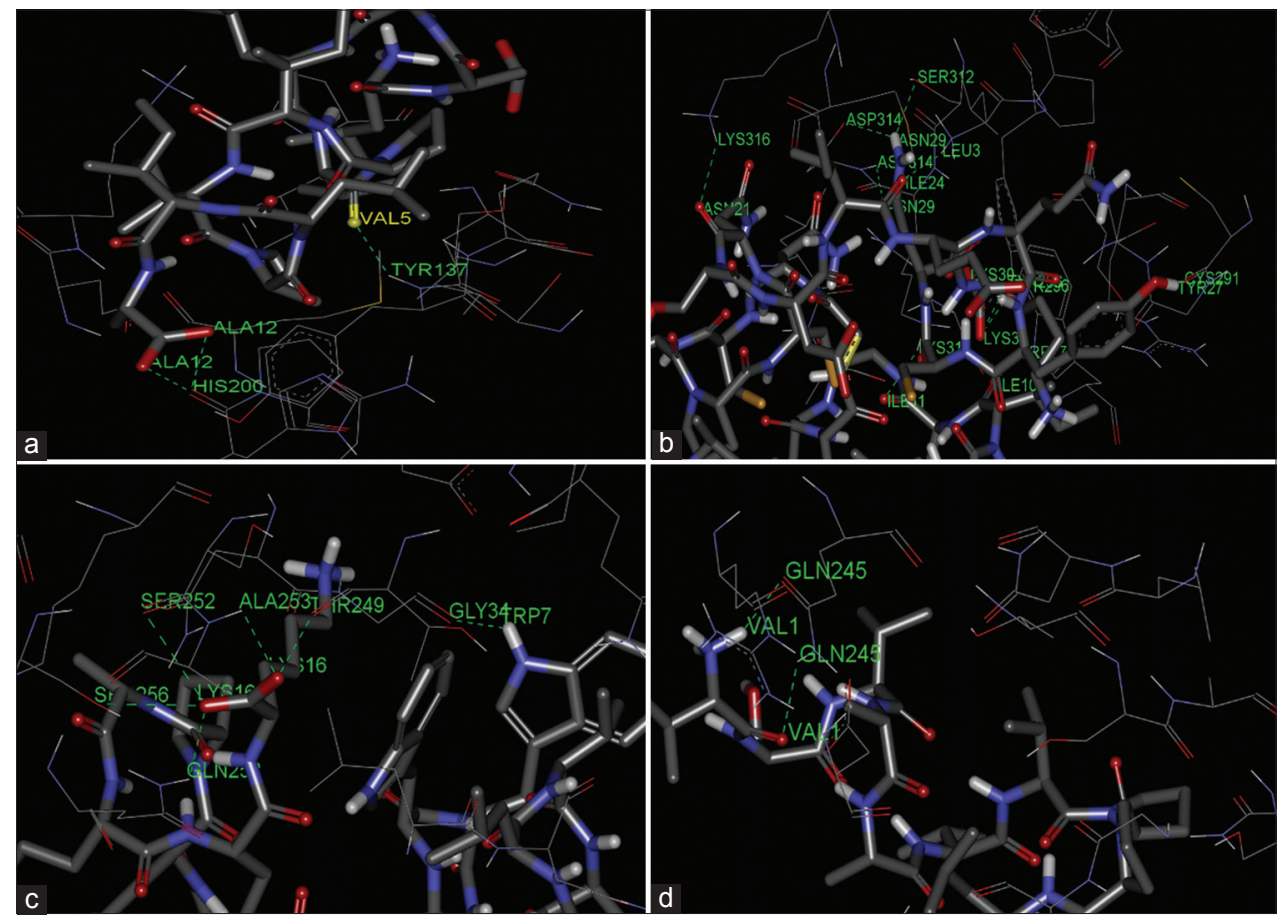

Fig. 1: Visualization of interacting amino acid residue after docking between receptor (1SDE) and peptide (a-d) using discovery studio 2.5 


\section{ACKNOWLEDGMENT}

The authors are highly thankful to the Ministry of Human Resource Development, Government of India and Department of Bioinformatics, MANIT, Bhopal, India, for providing support in the form of bioinformatics infrastructure facility to carry out this work.

\section{AUTHORS CONTRIBUTION:}

Sunil Kumar Suryawasnhi designed and performed experiments, analysed data and wrote the paper; and Usha Chouhan participate in drafting the article and revising it critically for important intellectual content.

\section{CONFLICTS OF INTERESTS}

None

\section{REFERENCES}

1. Anaya-López JL, López-Meza JE, Ochoa-Zarzosa A. Bacterial resistance to cationic antimicrobial peptides. Crit Rev Microbiol 2013;39:180-95.

2. Dutta P, Das S. Mammalian antimicrobial peptides: Promising therapeutic targets against infection and chronic inflammation. Curr Top Med Chem 2016;16:99-129.

3. Andreu D, Rivas L. Animal antimicrobial peptides: An overview. Biopolymers 1998;47:415-33.

4. Bastos P, Trindade F, Ferreira R, Casteleiro MA, Stevens R, Klein J, et al. Unveiling antimicrobial peptide-generating human proteases using PROTEASIX. J Proteomics 2018;171:53-62.

5. Singh S, Malhotra M, Majumdar DK. Antibacterial activity of Ocimum sanctum L. Fixed oil. Indian J Exp Biol 2005;43:835-7.

6. Singh V, Verma O. Ocimum sanctum (tulsi): Bio-pharmacological activities. WebmedCentral Pharmacol 2010;1:WMC001046.
7. Patil RS, Kokate MR, Kolekar SS. Bioinspired synthesis of highly stabilized silver nanoparticles using Ocimum tenuiflorum leaf extract and their antibacterial activity. Spectrochim Acta A Mol Biomol Spectrosc 2012;91:234-8.

8. Pratt RF. Substrate specificity of bacterial DD-peptidases (penicillinbinding proteins). Cell Mol Life Sci 2008;65:2138-55.

9. Silvaggi NR, Kaur K, Adediran SA, Pratt RF, Kelly JA. Toward better antibiotics: Crystallographic studies of a novel class of DD-peptidase/ beta-lactamase inhibitors. Biochemistry 2004;43:7046-53.

10. Gasteiger E, Hoogland C, Gattiker A, Se D, Wilkins MR, Appel RD, et al. Protein Identification and Analysis Tools on the ExPASy Server. New Jersey, NJ: Springer; 2005.

11. Waghu FH, Gopi L, Barai RS, Ramteke P, Nizami B, Idicula-Thomas S, et al. CAMP: Collection of sequences and structures of antimicrobial peptides. Nucleic Acids Res 2014;42:D1154-8.

12. Shen Y, Maupetit J, Derreumaux P, Tufféry P. Improved PEP-FOLD approach for peptide and miniprotein structure prediction. J Chem Theory Comput 2014;10:4745-58.

13. Kaushik V, Chauhan G, Singh J. In silico peptide based vaccine design against non-structural protein 5 of hepatitis $\mathrm{C}$ virus. Int J Pharm Pharm Sci 2013;6:82-3

14. Nisha N, Sreekumar S, Biju C. Identification of lead compounds with cobra venom detoxification activity in Andrographis Paniculata (Burm. F.) Nees through in silico method. Int J Pharm Pharm Sci 2016;8:212-7.

15. Schneidman-Duhovny D, Inbar Y, Nussinov R, Wolfson HJ. PatchDock and symmDock: Servers for rigid and symmetric docking. Nucleic Acids Res 2005;33:W363-7.

16. Mashiach E, Schneidman-Duhovny D, Andrusier N, Nussinov R, Wolfson HJ. FireDock: A web server for fast interaction refinement in molecular docking. Nucleic Acids Res 2008;36:W229-32.

17. Ritchie D, Orpailleur T. Hex 8.0. 0 User Manual; 1996.

18. Studio D. Version 2.5. San Diego, CA: Accelrys. Inc.; 2009. p. 92121.

19. Dziuba B, Dziuba M. New milk protein-derived peptides with potential antimicrobial activity: An approach based on bioinformatic studies. Int J Mol Sci 2014;15:14531-45. 